\title{
Amplification and Suppression of Traveling Waves along the Mouse Organ of Corti: Evidence for Spatial Variation in the Longitudinal Coupling of Outer Hair Cell-Generated Forces
}

\author{
미ames B. Dewey, ${ }^{1} \oplus^{\circledR B}$ Brian E. Applegate, ${ }^{2}$ and $\odot$ John S. Oghalai ${ }^{1}$ \\ ${ }^{1}$ The Caruso Department of Otolaryngology-Head and Neck Surgery, University of Southern California, Los Angeles, California 90033, and ${ }^{2}$ Department of \\ Biomedical Engineering, Texas A\&M University, College Station, Texas 77843
}

\begin{abstract}
Mammalian hearing sensitivity and frequency selectivity depend on a mechanical amplification process mediated by outer hair cells $(\mathrm{OHCs})$. $\mathrm{OHCs}$ are situated within the organ of Corti atop the basilar membrane (BM), which supports sound-evoked traveling waves. It is well established that OHCs generate force to selectively amplify BM traveling waves where they peak, and that amplification accumulates from one location to the next over this narrow cochlear region. However, recent measurements demonstrate that traveling waves along the apical surface of the organ of Corti, the reticular lamina (RL), are amplified over a much broader region. Whether $\mathrm{OHC}$ forces accumulate along the length of the RL traveling wave to provide a form of "global" cochlear amplification is unclear. Here we examined the spatial accumulation of RL amplification. In mice of either sex, we used tones to suppress amplification from different cochlear regions and examined the effect on RL vibrations near and far from the traveling-wave peak. We found that although OHC forces amplify the entire RL traveling wave, amplification only accumulates near the peak, over the same region where BM motion is amplified. This contradicts the notion that RL motion is involved in a global amplification mechanism and reveals that the mechanical properties of the $\mathrm{BM}$ and organ of Corti tune how $\mathrm{OHC}$ forces accumulate spatially. Restricting the spatial buildup of amplification enhances frequency selectivity by sharpening the peaks of cochlear traveling waves and constrains the number of OHCs responsible for mechanical sensitivity at each location.
\end{abstract}

Key words: basilar membrane; cochlear amplification; reticular lamina; traveling wave

Significance Statement

Outer hair cells generate force to amplify traveling waves within the mammalian cochlea. This force generation is critical to the ability to detect and discriminate sounds. Nevertheless, how these forces couple to the motions of the surrounding structures and integrate along the cochlear length remains poorly understood. Here we demonstrate that outer hair cell-generated forces amplify traveling-wave motion on the organ of Corti throughout the wave's extent, but that these forces only accumulate longitudinally over a region near the wave's peak. The longitudinal coupling of outer hair cell-generated forces is therefore spatially tuned, likely by the mechanical properties of the basilar membrane and organ of Corti. Our findings provide new insight into the mechanical processes that underlie sensitive hearing.

\section{Introduction}

Sound stimulation of the mammalian inner ear evokes a displacement wave on the basilar membrane (BM) that travels from the cochlear base to apex. Each longitudinal BM location is tuned to a different characteristic frequency (CF), such that highfrequency sounds elicit waves that peak near the base, and low

\footnotetext{
Received Oct. 3, 2018; revised Jan. 6, 2019; accepted Jan. 9, 2019.

Author contributions: J.B.D., B.E.A., and J.S.0 edited the paper; J.B.D., B.E.A., and J.S.0. designed research; J.B.D. performed research; J.B.D. analyzed data; J.B.D. and J.S.O. wrote the paper.

This work was supported by National Institutes of Health/National Institute on Deafness and Other Communication Disorders Grants R01 DC014450 and R01 DC013774 (J.S.0.) and F32 DC016211 (J.B.D.). We thank Drs. Christopher Shera and Karolina Charaziak for their comments on the paper.
}

frequencies elicit waves that peak more apically (Békésy, 1960). As the wave propagates, its magnitude is amplified by forces generated by the outer hair cells (OHCs) within the organ of Corti (Oghalai, 2004; Ashmore et al., 2010; Fig. 1A-C). Amplification of BM motion becomes apparent a short distance basal to the wave's peak and accumulates spatially as the wave travels over the amplifying region (Russell and Nilsen, 1997; Rhode and Recio, 2000; Ren, 2002). The gain at the peak is therefore partially in-

\footnotetext{
The authors declare no competing financial interests.

Correspondence should be addressed to John S. Oghalai at john.oghalai@med.usc.edu.

https://doi.org/10.1523/JNEUROSCI.2608-18.2019
}

Copyright $\odot 2019$ the authors $\quad 0270-6474 / 19 / 391805-12 \$ 15.00 / 0$ 


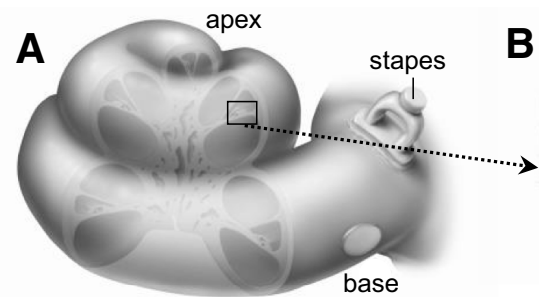

\section{BM: amplification builds up within amplifying region}

C
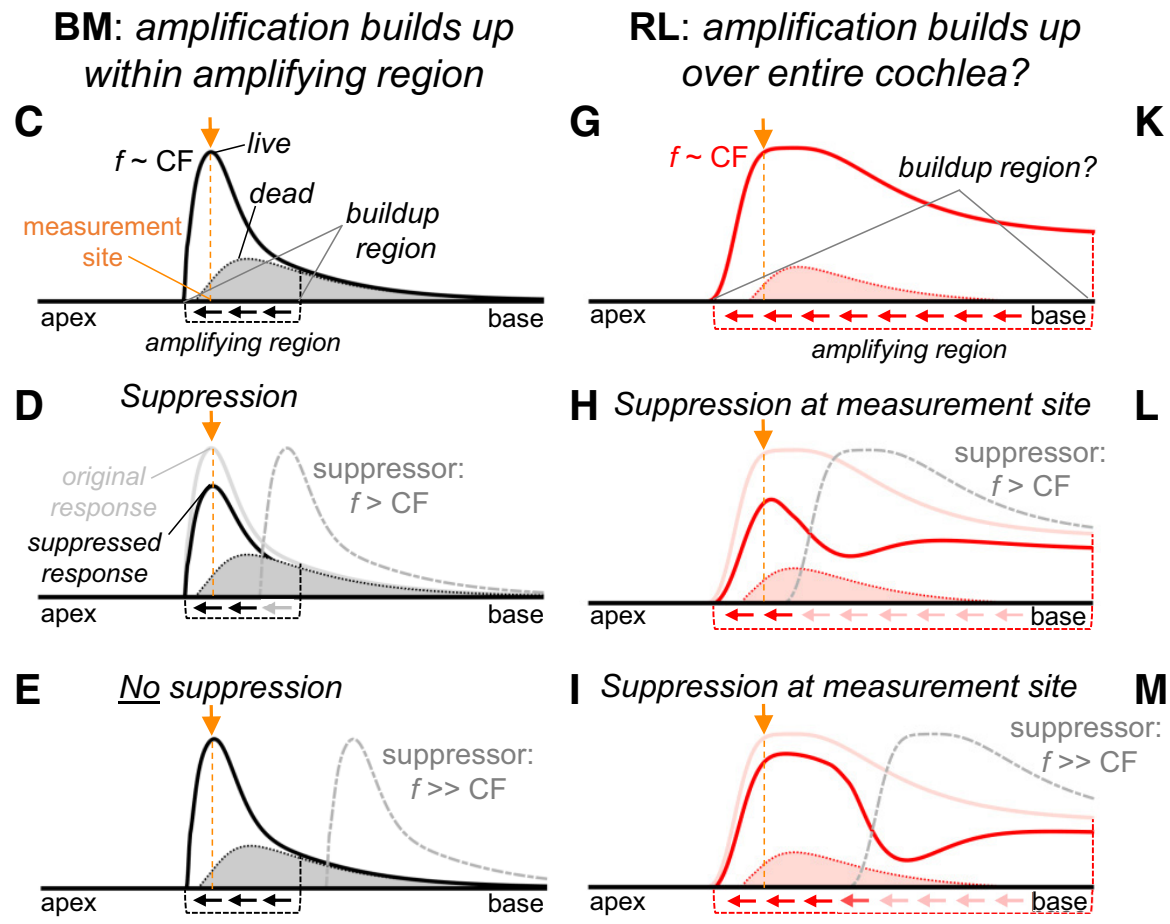

K
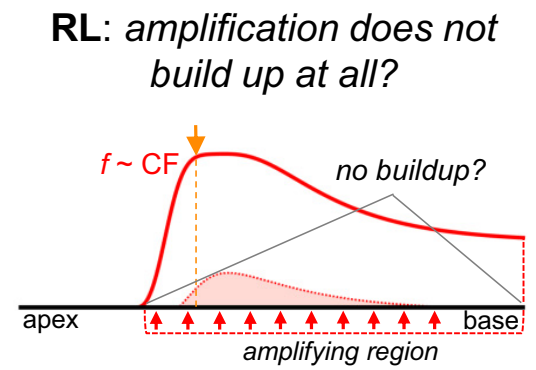

L No suppression at measurement site

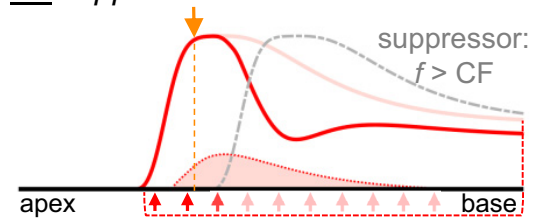

No suppression at measurement site

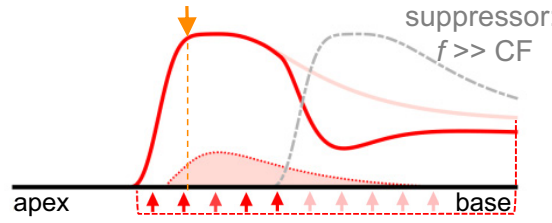

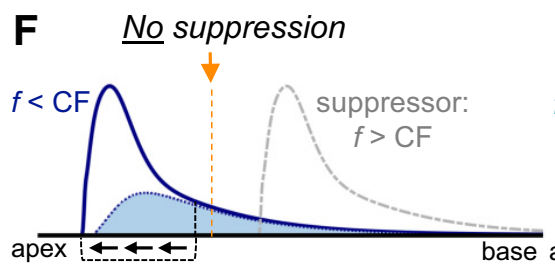

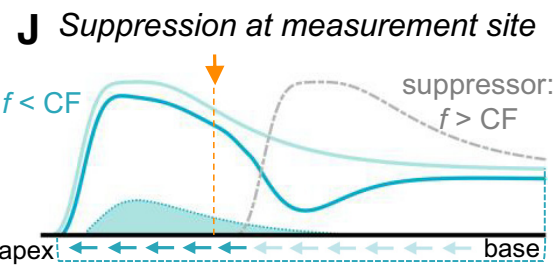

$\mathbf{N}$ No suppression at measurement site

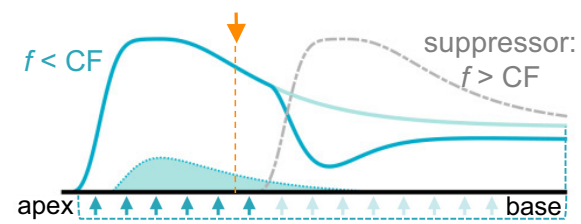

Figure 1. Assessing the spatial buildup of amplification in the mouse cochlea using suppression. $A$, Diagram indicating the approximate location where we measured vibrations in the apical turn of the mouse cochlea. $\boldsymbol{B}$, Cross-sectional diagram of the cochlear partition. The RL forms the apical surface of the $\mathrm{OHCs}$ and IHCs, which are coupled to the BM by various supporting cells. The overlying tectorial membrane (TM) is connected to the RL via the tallest row of $\mathrm{OHC}$ stereocilia. C, Diagram illustrating the spatial envelope of BM displacement along the length of the uncoiled cochlea. The response is shown for a stimulus frequency $(f)$ near the $\mathrm{CF}(\sim 9 \mathrm{kHz})$ of our measurement site (orange dashed line) in an active, live cochlea (solid line) and a passive, dead one (dotted line/shaded region). BM responses are amplified in the region where live and dead responses diverge (dashed bracket) and amplification builds up over this region (indicated by apically-pointing arrows). $\boldsymbol{D}-\boldsymbol{F}$, Known effects of suppressor tones on BM responses to near-CF $(\boldsymbol{D}, \boldsymbol{E})$ and below- $C F(\boldsymbol{F})$ tones. Near-CF responses are reduced by above-CF suppressor tones that excite the amplifying/buildup region (gray line, with $f>(F ; D)$ but not by suppressor tones that only excite more basal regions (with $f \gg(F ; E$ ). Below-CF responses are not reduced because there is no amplification to suppress at our measurement site $(\boldsymbol{F})$. $\boldsymbol{G}$, Envelope of the RL response to a near-CF tone in a live and dead cochlea, illustrating that RL motion is amplified throughout the traveling wave. Apically-pointing arrows indicate the hypothetical scenario in which amplification builds up throughout the amplifying region. $\boldsymbol{H}-\mathbf{J}$, Predictions for the suppression of RL responses if amplification builds up throughout the amplifying region. At our measurement site, RL responses to both near- $C F(H, I)$ or below-CF $(J)$ tones should be reduced by suppressors that excite any portion of the amplifying region, including those that excite regions far basal to the peak. $\boldsymbol{K}-\boldsymbol{N}$, Diagrams illustrating the alternate scenario in which amplification of the RL traveling wave does not build up at all (K). RL motion at any given location would therefore only be amplified by local OHC-generated forces (vertically-oriented arrows indicate local amplification). At our measurement site, RL responses to near-CF ( $L$, $M$ ) or below-CF $(\boldsymbol{N})$ tones should not be reduced by suppressor tones that do not excite this location.

herited from OHC activity at more basal locations, and damaging or disturbing OHCs anywhere within the amplifying region can reduce the wave's peak magnitude (Cody, 1992; Rhode, 2007; Fisher et al., 2012; Versteegh and van der Heijden, 2013). The buildup of BM amplification over a restricted region is thought to underlie the high sensitivity and sharp frequency tuning of responses measured from inner hair cells (IHCs), the primary afferent receptors, and auditory nerve fibers in high-frequency regions of the mammalian cochlea (Patuzzi and Robertson, 1988; Narayan et al., 1998).

Recent measurements, however, demonstrate that travelingwave motion on the reticular lamina (RL), at the apical surface of the OHCs, is quite different from BM motion (Lee et al., 2016; Ren et al., 2016b; Cooper et al., 2018). RL traveling waves are amplified over a much broader spatial region, perhaps extending throughout the cochlear base. At a given location, RL motion is 
therefore not just amplified at the CF, but also at frequencies several octaves lower. These new data suggest that BM motion provides an incomplete description of cochlear mechanics and raise important questions regarding the functional role of below-CF RL amplification. Although it has been proposed that amplification of the RL traveling wave distant from the peak underlies a global hydrodynamic mechanism for building up the wave as it propagates (Ren et al., 2016b; He et al., 2018), the buildup of RL amplification has not yet been assessed.

Here we examined how RL traveling-wave amplification builds up in the mouse cochlear apex. To do so, we studied how tone-evoked BM and RL vibrations were suppressed by the presentation of a second tone. Suppression occurs when the response to one tone saturates $\mathrm{OHC}$ mechanotransduction currents, thus limiting OHC force generation (Geisler et al., 1990). By varying the suppressor tone frequency, we selectively reduced amplification from different cochlear locations, allowing us to map the region where amplification accumulates (which we term the "buildup region"). Previous measurements have shown that the BM traveling-wave peak can be suppressed by tones that excite any region where the wave is amplified, consistent with amplification building up over the amplifying region (Rhode, 2007; Versteegh and van der Heijden, 2013; Fig. $1 C-F$ ). If this also holds true for the RL traveling wave, RL amplification would build up over the entire cochlea, because the RL's amplifying region extends to the cochlear base. RL motion near or distant from the peak should be reduced by a wide range of suppressor frequencies, including those that stimulate locations far basal to the peak (Fig. 1G-J). If the extreme opposite scenario were true and RL amplification did not build up at all, RL motion should only be suppressed by tones that stimulate OHCs at the measurement site (Fig. $1 K-N$ ).

Interestingly, our measurements reveal an intermediate scenario in which RL traveling-wave amplification primarily builds up near the wave's peak, with a buildup region similar to that observed for the BM. These data suggest that the coupling of $\mathrm{OHC}$-generated forces along the RL and BM is spatially tuned, likely by the mechanical properties of the BM and organ of Corti.

\section{Materials and Methods}

Mouse preparation. We measured cochlear vibrations in vivo in adult (4to 6-week-old) wild-type CBA/CaJ mice (stock \#000654, The Jackson Laboratory) of either sex $(n=10 ; 5$ male). All procedures were approved by the Institutional Animal Care and Use Committee at the University of Southern California. Mice were anesthetized with ketamine (80-100 mg/ $\mathrm{kg}$ ) and xylazine (5-10 $\mathrm{mg} / \mathrm{kg}$ ) and positioned on a heating pad to maintain a core body temperature of $\sim 37^{\circ} \mathrm{C}$. Supplemental doses of anesthesia were administered throughout the experiment to ensure areflexia. The top of the skull was exposed and glued to a custom headholder using dental cement, and a ventrolateral approach was used to surgically access the left middle ear bulla. The bone below the tympanic ring was then carefully chipped away so that the top of the otic capsule could be visualized. The pinna was removed and the external ear canal was partially dissected away to allow positioning of the tip of a probe containing both a microphone and sound sources (ER10X, Etymotic Research) within a few $\mathrm{mm}$ of the tympanic membrane. The probe tip was glued to the rim of the dissected ear canal to create a closed sound field. After completing all desired vibration measurements, mice were killed by anesthetic overdose and a limited set of measurements was repeated postmortem.

Volumetric optical coherence tomography and vibrometry. We used an optical coherence tomography-based approach [volumetric optical coherence tomography and vibrometry (VOCTV)] to noninvasively image through the cochlear bone and record vibratory responses from the apical cochlear turn, as described previously (Gao et al., 2014; Lee et al.,
2015, 2016). Briefly, the custom-built system consisted of a broadband swept-source laser (MEMS-VCSEL, Thorlabs; center-wavelength = $1300 \mathrm{~nm}$, bandwidth $=100 \mathrm{~nm}$, sweep rate $=200 \mathrm{kHz}$ ), a dual-balanced photodetector (WL-BPD600MA, Wieserlabs), and a high-speed digitizer (NI-5761, National Instruments) connected to a desktop PC. An adaptor attached to the bottom of the dissecting microscope (Stemi-2000, Zeiss) housed a 2-D voice coil mirror that was used to scan the light source across the preparation to obtain cross-sectional images of the cochlear duct. After imaging the apical cochlear turn, vibratory responses were obtained from specific voxels on the BM and from the RL, near the top of the $\mathrm{OHC}$ region. Measurements were always obtained from local maxima in the reflectivity versus depth profile to increase the vibratory signal-to-noise ratio. The angle between the light source and the BM was $\sim 60-75^{\circ}$, such that the vibration measurements largely captured the transverse motions of the BM and RL.

The imaging resolution of our system was previously determined to be $\sim 9.8$ and $\sim 11.4 \mu \mathrm{m}$ (full-width at half-maximum) in the lateral and axial dimensions, respectively (Lee et al., 2015, 2016). Because the BM is $\sim 20 \mu \mathrm{m}$ thick in the mouse cochlear apex (Keiler and Richter, 2001), our measurements of BM motion were likely dominated by the BM. However, because we cannot measure vibrations directly from the surface of the RL, our measurements of RL vibration probably reflect the combined motion of the RL and the upper half of the OHC region, over the width of at least one OHC. OHCs in the mouse cochlear apex are $\sim 20 \mu \mathrm{m}$ in length, $\sim 5-7 \mu \mathrm{m}$ in diameter, and spaced $\sim 8 \mu \mathrm{m}$ apart (Zajic and Schacht, 1987; Soons et al., 2015). We did not systematically explore the vibratory properties of locations deeper within the organ of Corti, where the motions are likely more complex, with transverse, radial, and longitudinal components (Gao et al., 2014; Lee et al., 2016; Cooper et al., 2018). However, to the extent that the motion of these locations is influenced by OHC activity (Cooper et al., 2018), measurements of two-tone suppression at these locations would presumably be similar to what we measured at the RL.

Stimulus paradigms. Sound stimuli were calibrated before each experiment using a Brüel \& Kjær 1/8 inch microphone (Type 4138) coupled to the sound delivery probe via a $\sim 3 \mathrm{~mm}$ length of tubing to approximate the residual ear canal. At the beginning of an experiment, responses to 50-100 ms tones were obtained to determine the CF of the cochlear location being imaged. The $\mathrm{CF}$ was defined as the frequency at the peak of the $\mathrm{BM}$ response to a $30 \mathrm{~dB}$ sound pressure level (SPL) tone swept from 1 to $15 \mathrm{kHz}$ in $0.5 \mathrm{kHz}$ steps, and was either $9 \mathrm{kHz}(n=4)$ or $9.5 \mathrm{kHz}(n=$ 6 ) in the 10 mice studied. The effects of different suppressor tones on the response to a near-CF probe tone (with frequency $0.1 \mathrm{kHz}$ above the $\mathrm{CF}$, thus either 9.1 or $9.6 \mathrm{kHz}$ ) or low-frequency probe tone $(2.1-6.1 \mathrm{kHz})$ were then assessed. For each stimulus presentation, a probe tone was presented for $100 \mathrm{~ms}$, and a suppressor tone was presented for $100 \mathrm{~ms}$ after the probe tone had been on for $50 \mathrm{~ms}$. This yielded three $50 \mathrm{~ms}$ intervals containing the response to (1) the probe alone, (2) both the probe and suppressor tones, and (3) the suppressor alone. All responses were averaged across $4-8$ stimulus presentations. The response to the probe and/or suppressor frequency components in each interval was then determined from a fast Fourier transform after applying a Hanning window to the time-domain signal. Suppression was defined as the amount (in $\mathrm{dB}$ ) by which the probe response was reduced by the presence of the suppressor.

For each probe tone, suppression was examined as a function of the suppressor tone frequency (extending from the CF in $1 \mathrm{kHz}$ steps over a range covering $\sim 1-20 \mathrm{kHz}$ ) and level $(30-90 \mathrm{~dB}$ SPL in $5 \mathrm{~dB}$ steps). Suppression measurements were attempted for as many probe frequencies and levels as possible. However, the number of usable measurements from a given preparation was limited by the high measurement noise floors at low probe frequencies, and premature death resulting from the anesthesia. To facilitate comparisons across the same set of measurement conditions within a sufficient number of mice, we prioritized characterizing the suppression of $\mathrm{BM}$ and RL responses to near-CF probe tones and $\mathrm{RL}$ responses to $4.1 \mathrm{kHz}$ probe tones, using a probe level of $60 \mathrm{~dB}$ SPL, before obtaining measurements at other probe frequencies and levels. For a stimulus level of $60 \mathrm{~dB}$ SPL, BM and RL responses to near-CF tones and RL responses to $4.1 \mathrm{kHz}$ tones were similar in magnitude and 
had signal-to-noise ratios that were adequate for characterizing suppression. Suppression of BM responses to below-CF probe tones was not assessed, as there is little amplification of BM motion at these frequencies (Rhode, 2007; Versteegh and van der Heijden, 2013) and the vibratory amplitudes were too small to reliably characterize suppression.

Responses to single tones presented at $30 \mathrm{~dB}$ SPL were repeatedly assessed throughout each experiment to verify that the CF and sensitivity of the preparation did not change over time. Responses to single tones were also obtained after death so as to characterize the loss of OHCmediated amplification postmortem. However, suppression measurements were not repeated after death, because of the loss of the nonlinearities associated with cochlear amplification, as well as the reduction of most probe responses into the measurement noise floor.

Data analysis. For each probe frequency and level, the suppressor level required to reduce the probe response by a criterion amount (1.5-12 dB) was calculated for each suppressor frequency to produce a family of suppression threshold curves. Curves were parameterized so as to determine the frequency and threshold level at the minimum (or "tip") of the curve, as well as the sharpness of tuning, which was assessed by dividing the tip frequency by the bandwidth $10 \mathrm{~dB}$ up from the tip, yielding the quality factor $\mathrm{Q}_{10 \mathrm{~dB}}$. Most importantly, we assessed the high-frequency extent of suppression by determining the highest suppressor frequency that produced a criterion amount of suppression when the suppressor was presented at $70 \mathrm{~dB}$ SPL. In other words, we calculated the $70 \mathrm{~dB}$ SPL intercept for each suppression threshold curve at frequencies above the CF. Using the frequency-to-place map of the mouse cochlea (Müller et al., 2005), we then calculated the distance between the measurement location and the tonotopic place of the highest effective suppressor frequency (defined as the $70 \mathrm{~dB}$ SPL suppression threshold intercept). This yielded an estimate of the longitudinal region over which OHCs contributed amplification to the probe response at our measurement location. In some cases, calculation of the $70 \mathrm{~dB}$ SPL intercept required that suppression thresholds be extrapolated to slightly higher frequencies. To average responses across preparations with different CFs, stimulus frequencies were converted to octave values relative to the $\mathrm{CF}$, and the data from each mouse were interpolated in fixed 1/20th-octave steps. All vibratory data presented and analyzed in this report had magnitudes that were at least 3 SDs above the mean noise floor in frequency bins \pm $200-300 \mathrm{~Hz}$ around the stimulus frequency. Response phases were corrected by subtracting the stimulus phase measured in the ear canal.

Experimental design and statistical analysis. A within-subjects design was used to compare the vibratory characteristics of the BM and RL as well as the suppression of BM and RL responses to different probe frequencies and/or levels. Repeated-measures ANOVAs followed by post hoc pairwise comparisons with Bonferroni corrections were conducted using SPSS software to determine the statistical significance (at the $p<$ 0.05 level) of differences in suppression threshold parameters across measurement conditions. The Greenhouse-Geisser correction was used to adjust the degrees of freedom of the $F$ distribution in cases where the data violated the assumptions of sphericity. Paired, two-tailed $t$ tests were used when only two quantities were obtained from the same mice and compared. One-sample $t$ tests were also used to assess whether the tip frequencies of the suppression threshold curves were significantly different from the CF. For $t$ tests and ANOVAs, we provide the $t$ or $F$ statistic along with the degrees of freedom in parentheses (for ANOVAs, both between-group and within-group degrees of freedom are reported), the $p$ value, and the number of mice $(n)$ included in each test. The number of mice used in each analysis varied because it was not possible to obtain suppression measurements for all probe frequencies and levels in every preparation. All reported values are the average \pm SEM. When reported in the context of statistical comparisons across measurement conditions, averages only include data from mice in which all relevant measurement conditions were completed.

\section{Results}

\section{$B M$ and $R L$ responses to single tones}

We used VOCTV to noninvasively image through the cochlear bone (Fig. 2A,B) and record sound-evoked vibrations from the apical turn of the adult mouse cochlea (Gao et al., 2014; Lee et al.,
$2015,2016)$. We first characterized the vibrations of the BM and $\mathrm{RL}$ in response to single tones and determined the $\mathrm{CF}$ of a given measurement location (Fig. $2 C-F$; $\mathrm{CF}=9 \mathrm{kHz}$ in this example). As described previously (Lee et al., 2016; Ren et al., 2016b), displacements of the BM and RL exhibited nonlinear, compressive growth with increasing stimulus level for stimulus frequencies near the $\mathrm{CF}$, and more linear growth at lower frequencies. RL responses were generally larger and grew more compressively than BM responses, particularly at low frequencies. These differences are more evident after normalizing the displacements to the stimulus pressure (Fig. $2 G, H$ ), where the degree of nonlinearity is indicated by the separation between curves obtained at different stimulus levels. After death, the nonlinearity observed in BM and $\mathrm{RL}$ responses was eliminated, and low-frequency RL responses were dramatically reduced in magnitude. The vulnerability of low-frequency RL responses indicates that, at frequencies well below the CF, RL motion is actively amplified by OHCs. In contrast, $\mathrm{BM}$ motion is amplified very little at low frequencies. The mean live/dead displacement ratio for a $60 \mathrm{~dB}$ SPL $4 \mathrm{kHz}$ tone was $4.04 \pm 0.91 \mathrm{~dB}$ for the BM, and $23.82 \pm 0.75 \mathrm{~dB}$ for the RL (significantly different by paired $t$ test, $t_{(9)}=21.55, p<0.001$, $n=10)$.

Although measurements from the base of the mouse cochlea have shown that the RL moves out of phase with the BM at low frequencies and in phase near the CF (Ren et al., 2016b), we observed the opposite phase relationship (Fig. $2 I, J$ ). BM and RL motion were nearly in phase at low frequencies, and RL motion progressively lagged BM motion as the stimulus frequency was increased (RL-BM phase for a $70 \mathrm{~dB}$ SPL, CF tone $=-0.33 \pm$ 0.04 cycles; $t_{(8)}=8.59, p<0.001, n=9$, paired $t$ test). This phase difference was physiologically vulnerable and largely disappeared after death (Fig. 2J). The different RL-BM phase relationship observed in our measurements could possibly be attributed to the different measurement location and/or differences in measurement angle (Cooper et al., 2018).

\section{Suppression of responses to near-CF and below-CF tones}

We next examined how a second tone suppressed BM and RL responses to a primary tone (referred to as the "probe") presented at a frequency near the CF of the measurement site. This allowed us to map the extent of the buildup region basal to the peaks of the $\mathrm{BM}$ and RL traveling waves. Near-CF probes had frequencies 0.1 $\mathrm{kHz}$ above the CF, and thus were either 9.1 or $9.6 \mathrm{kHz}$. Representative data are shown from an experiment in which responses to a $60 \mathrm{~dB}$ SPL $9.6 \mathrm{kHz}$ probe were monitored while suppressor tones at different frequencies were varied in level (Fig. 3).

We found that BM and RL responses to near-CF probes were suppressed by a similar range of frequencies extending just above the CF (Fig. $3 D, E$ ). Suppressor tones with frequencies more than an octave above the CF (e.g., $20.5 \mathrm{kHz}$ ) produced little suppression $(<1 \mathrm{~dB})$ of either BM or RL responses, indicating that amplification at the BM or RL traveling-wave peak did not depend on $\mathrm{OHC}$-generated forces from far basal regions. However, suppressor tones with frequencies slightly above the CF (e.g., 14.5 $\mathrm{kHz}$ ) did reduce near-CF probe responses, suggesting that amplification built up from regions just basal to the measurement site. Suppression caused by a $14.5 \mathrm{kHz}$ tone was small $(<5 \mathrm{~dB})$ and grew little with increasing suppressor level $(<0.1 \mathrm{~dB} / \mathrm{dB})$. This is consistent with the notion that the wave evoked by the $14.5 \mathrm{kHz}$ tone only partly overlapped with the buildup region and grew compressively with stimulus level near its peak. Near-CF suppressor tones (e.g., $9.5 \mathrm{kHz}$ ) were most effective at reducing the probe response (with suppression approaching $\sim 30 \mathrm{~dB}$ ), 
A
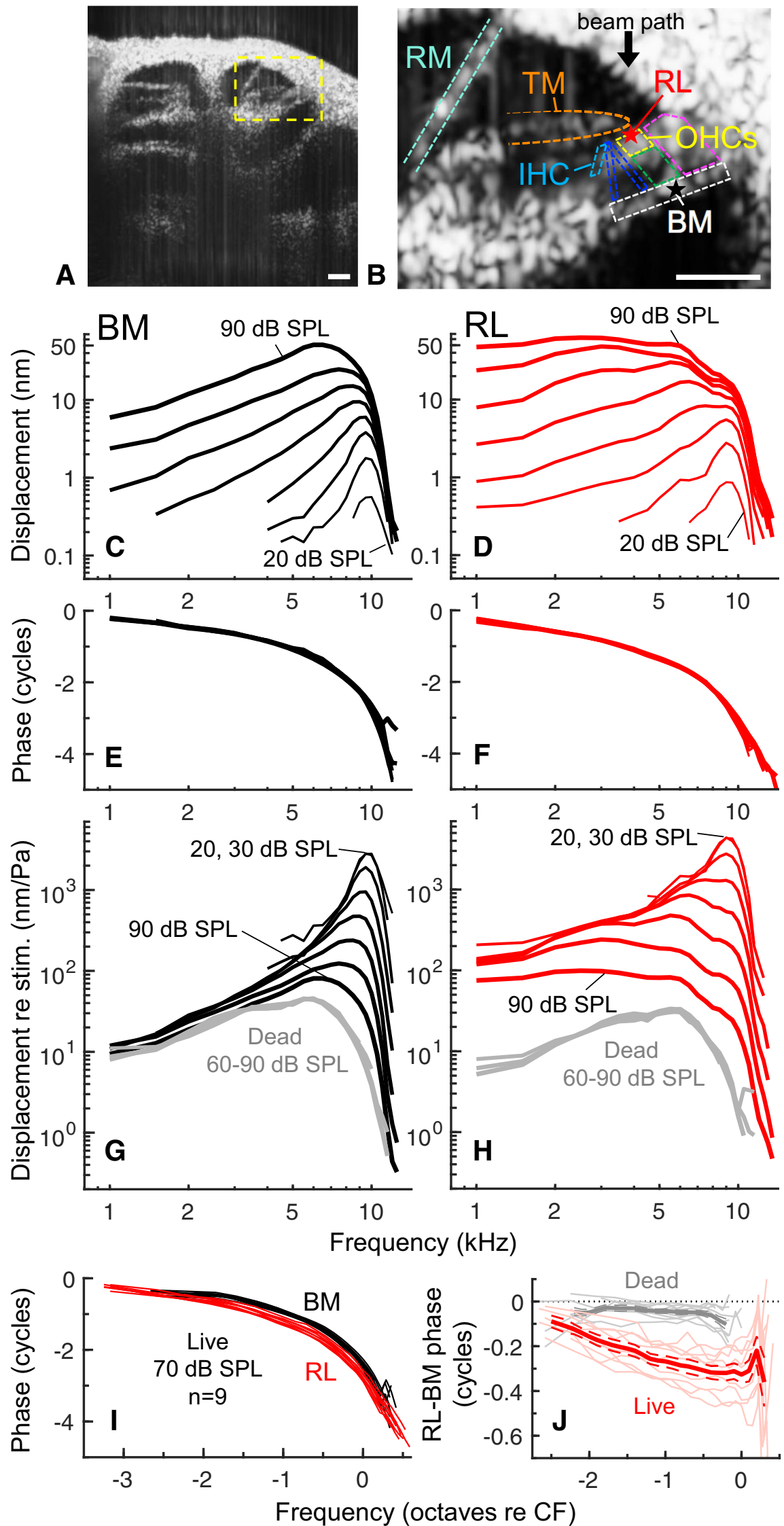

Figure 2. Single-tone responses of the BM and RL. $\boldsymbol{A}$, Cross-sectional image of a mouse cochlea obtained in vivo with VOCTV. The measurement location in the apical turn is highlighted. Scale bar, $100 \mu \mathrm{m}$. $\boldsymbol{B}$, Magnification of highlighted region from image in $\boldsymbol{A}$ with the locations of the BM, TM, Reissner's membrane (RM), and various cellular regions within the organ of Corti outlined. Outlined but not labeled are the pillar cells (in blue), which bound the tunnel of Corti; the Deiters' cells (in green), which support the whereas below-CF suppressors (e.g., 3.5 $\mathrm{kHz}$ ) produced comparable reductions only at high suppressor levels $(>70 \mathrm{~dB}$ SPL). These patterns can be attributed to the greater sensitivity and more compressive growth of responses to near-CF versus below-CF tones at the measurement site (Ruggero et al., 1992; Cooper, 1996; Rhode, 2007).

The above results suggest that amplification of the RL traveling-wave peak does not build up from far basal regions. We therefore next directly assessed the buildup of RL amplification basal to the peak by examining the suppression of RL responses to a $4.1 \mathrm{kHz}$ probe tone. This below-CF tone elicited a wave that peaked apical to our measurement site, allowing us to study a more basal region of the RL traveling wave (Fig. $3 C$ ). In contrast to $\mathrm{BM}$ and $\mathrm{RL}$ responses to near-CF probes, we found that RL responses to the $4.1 \mathrm{kHz}$ probe were only suppressed by tones that directly vibrated the measurement site (e.g., 3.5 and $9.5 \mathrm{kHz}$ ), and were not influenced by suppressor frequencies much

$\mathrm{OHCs}$; and the lateral supporting cell region (in magenta), which include Hensen's, Claudius', and Boettcher's cells. Stars indicate locations on the BM and RL where vibrations were measured. Scale bar, $100 \mu \mathrm{m}$. C, D, Sound-evoked displacements of the BM $(\boldsymbol{C})$ and $\mathrm{RL}(\boldsymbol{D})$ obtained from a representative mouse at a location with a CF of $9 \mathrm{kHz}$. Displacement magnitudes are plotted as a function of the stimulus tone frequency (1-15 kHz in $0.5 \mathrm{kHz}$ steps) for different stimulus levels (20 -90 dB SPL, $10 \mathrm{~dB}$ steps). Curves for the lowest and highest stimulus levels are labeled. For clarity, displacements were smoothed across frequency with a three-point moving average. $\boldsymbol{E}, \boldsymbol{F}$, Phase of BM $(\boldsymbol{E})$ and RL $(\boldsymbol{F})$ displacements as a function of frequency. Curves for different stimulus levels largely overlap at the scale shown. Increasing phase lags with increasing stimulus frequency indicate traveling-wave propagation. $\boldsymbol{G}, \boldsymbol{H}$, Displacements of the BM $(\boldsymbol{G})$ and $\mathrm{RL}(\boldsymbol{H})$ normalized to the evoking stimulus pressure in Pascals, revealing frequency-and level-dependent response nonlinearities. After death, BM and $\mathrm{RL}$ responses near the CF were dramatically reduced and nonlinearities were eliminated (overlapping gray curves were obtained for $60-90 \mathrm{~dB}$ SPL tones). RL responses below the CF were also reduced postmortem at all stimulus levels. $I$, Phase of $B M$ and RL responses to $70 \mathrm{~dB}$ SPL tones for all live mice in which single-tone responses were obtained with sufficient frequency resolution at this stimulus level. Response phases were consistent across preparations, and reveal that RL motion progressively lagged BM motion with increasing stimulus frequency. The frequency axis is expressed in octaves relative to the $\mathrm{C} F$ to facilitate comparison across mice.J, The difference between the BM and RL response phases shown in $I$, as well as the RL-BM phase difference in the same mice after death, demonstrating that it was physiologically vulnerable. Individual and average data are shown with thin and thick lines, respectively (dashed lines indicate \pm SEM). Individual data falling below the measurement noise floor are not shown. Average values are only shown for frequencies where data from at least five mice exceeded the noise floor. 
A BM: near-CF probe $(9.6 \mathrm{kHz})$
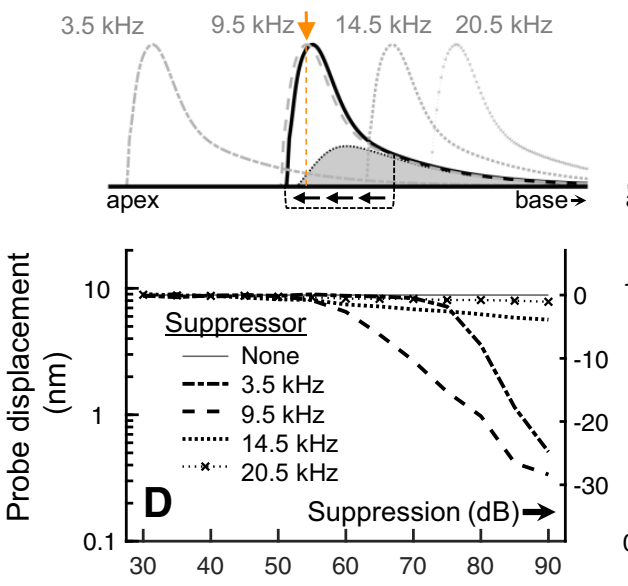

BM: near-CF probe $(9.6 \mathrm{kHz})$
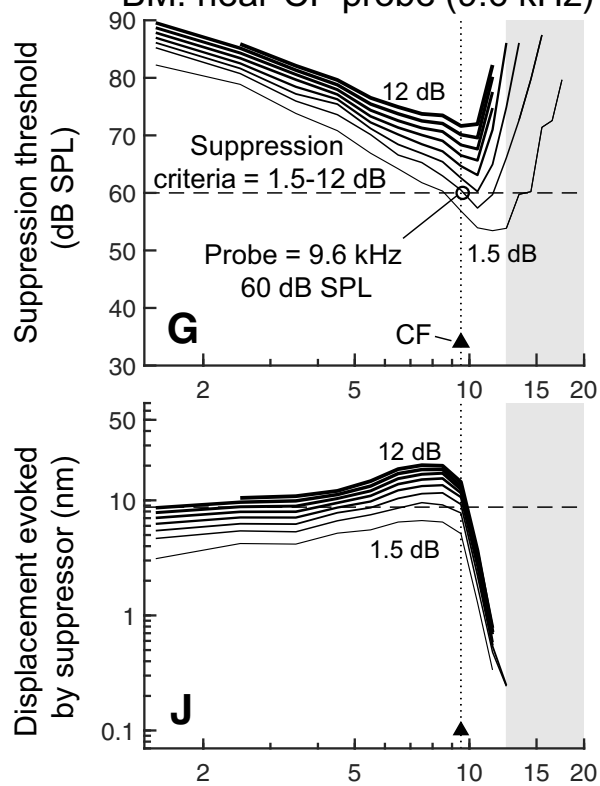

B RL: near-CF probe $(9.6 \mathrm{kHz})$

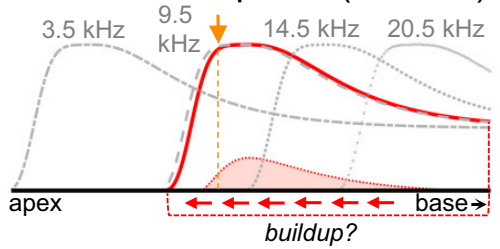

[ $20.5 \mathrm{kHz}$

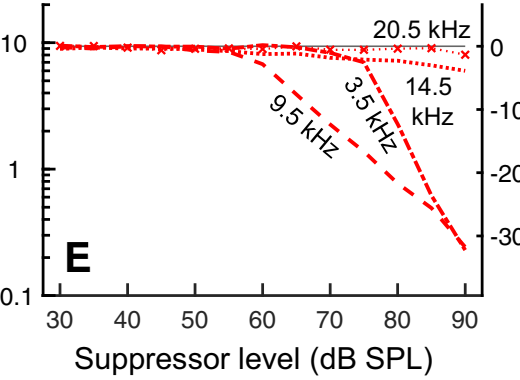

$\mathrm{RL}$ : near-CF probe $(9.6 \mathrm{kHz})$
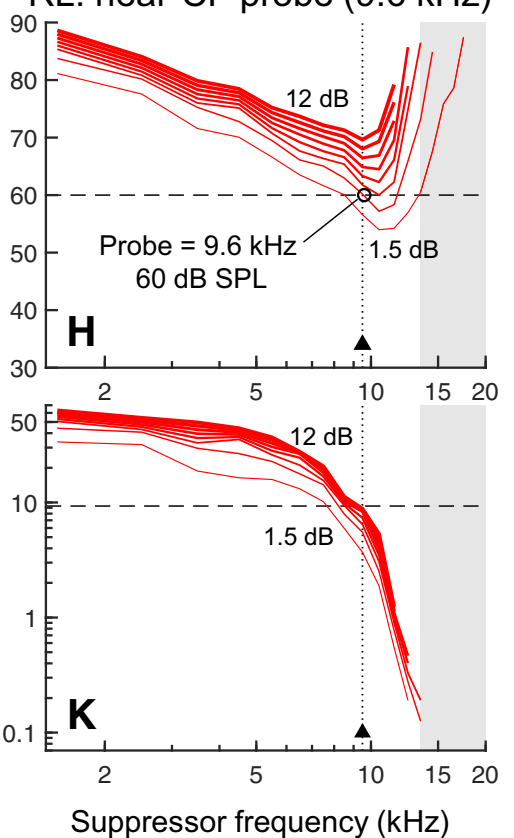

C RL: below-CF probe $(4.1 \mathrm{kHz})$
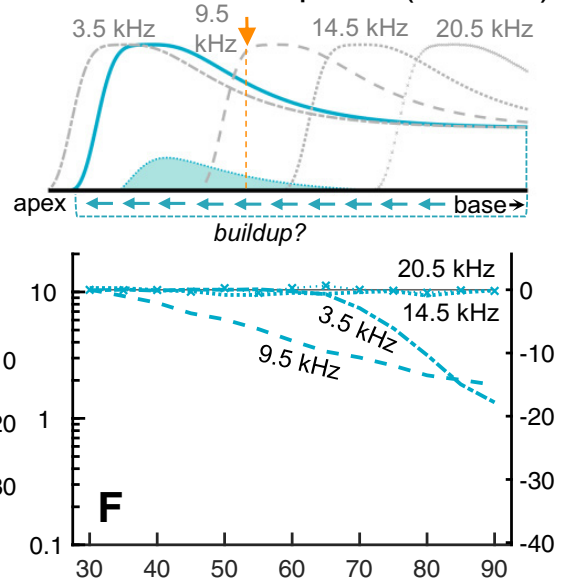

$\mathrm{RL}$ : below-CF probe $(4.1 \mathrm{kHz})$
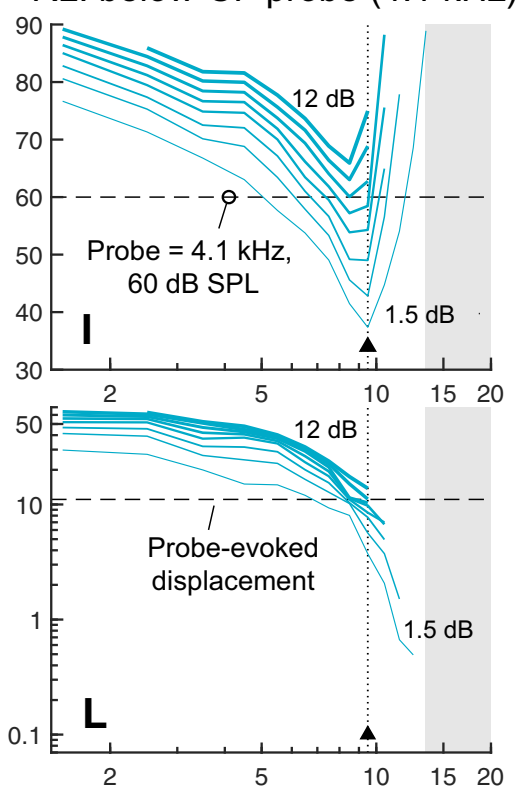

Figure 3. Suppression of responses to near-CF and below-CF probe tones. $\boldsymbol{A}-\boldsymbol{C}$, Diagrams illustrating the waves elicited by a probe tone fixed at a frequency near the $C$ F of the measurement site (here $9.5 \mathrm{kHz}$ ) for the BM $(\boldsymbol{A})$ and $\mathrm{RL}(\boldsymbol{B})$, or at a below-CF frequency for the RL (C). The waves elicited by suppressor tones at different frequencies are shown in gray. $\boldsymbol{D}, \boldsymbol{E}$, Representative BM (D) and $\mathrm{RL}(\boldsymbol{E})$ displacements evoked by a near-CF probe tone ( $9.6 \mathrm{kHz}, 60 \mathrm{dBSPL})$ in the absence (thin, solid line) or presence of suppressor tones at frequencies below, at, or above the CF (see legend), plotted as a function of suppressor level. Right axes indicate the amount of suppression in $\mathrm{dB}$ relative to the unsuppressed probe response. For clarity, the unsuppressed probe response was averaged across all measurement conditions. Responses in the presence of each suppressor (in $\mathrm{nm}$ ) were rescaled appropriately using the amount of suppression (in $\mathrm{dB}$ ) observed in each condition. $\boldsymbol{F}$, RL displacements for a below- $\mathrm{CF}(4.1 \mathrm{kHz})$ probe tone in the absence and presence of the same suppressor tone frequencies. The probe response was relatively insensitive to above-CF suppressor tones, indicating that amplification of the response did not build up from locations basal to the measurement site. G-I, Suppressor tone levels required to achieve fixed amounts of suppression, ranging from 1.5-12 dB in $1.5 \mathrm{~dB}$ steps, as a function of suppressor tone frequency (data are from the same preparation as in $\boldsymbol{D}-\boldsymbol{F}$ ). The $(\boldsymbol{F}$ is indicated by the triangle/dotted vertical line; dashed horizontal line and circle indicate the probe frequency and level. Gray shaded regions indicate the range of suppressor frequencies for which the suppressor evoked no displacement above the measurement noise floor $(\sim 0.1 \mathrm{~nm})$. $\boldsymbol{J}-\mathbf{L}$, Displacements elicited by the suppressor tone at the threshold for each suppression criterion shown in $\mathbf{G}-\mathbf{I}$. Suppressor-evoked displacements falling below the measurement noise floor are not shown. Dashed horizontal line indicates the unsuppressed response to the probe tone.

higher than the CF (14.5 or $20.5 \mathrm{kHz}$; Fig. $3 F)$. The amplification of RL responses to the $4.1 \mathrm{kHz}$ probe therefore did not build up significantly from regions basal to our measurement site, confirming that the buildup of amplification is restricted to a region near the peak of the RL traveling wave.

\section{Estimating the extent of the buildup regions for the BM} and RL

To more precisely estimate the spatial extent of the buildup regions for BM and RL responses, we calculated the suppressor level required to reduce the probe response by a criterion amount for a wide range of suppressor frequencies (Fig. $3 G-I$ ). Suppression thresholds are shown for criteria of 1.5-12 dB, demonstrating the consistency of the patterns across criteria. For BM and RL responses to near-CF probes, small amounts of suppression were most effectively achieved with suppressor frequencies just above the CF and were observed at frequencies up to $17.5 \mathrm{kHz}(0.88$ octaves above the CF). Suppression was observed despite the fact that suppressor tones above $\sim 13 \mathrm{kHz}$ did not elicit displacements above the noise floor at the measurement site (Fig. $3 G, H, J, K$, gray 

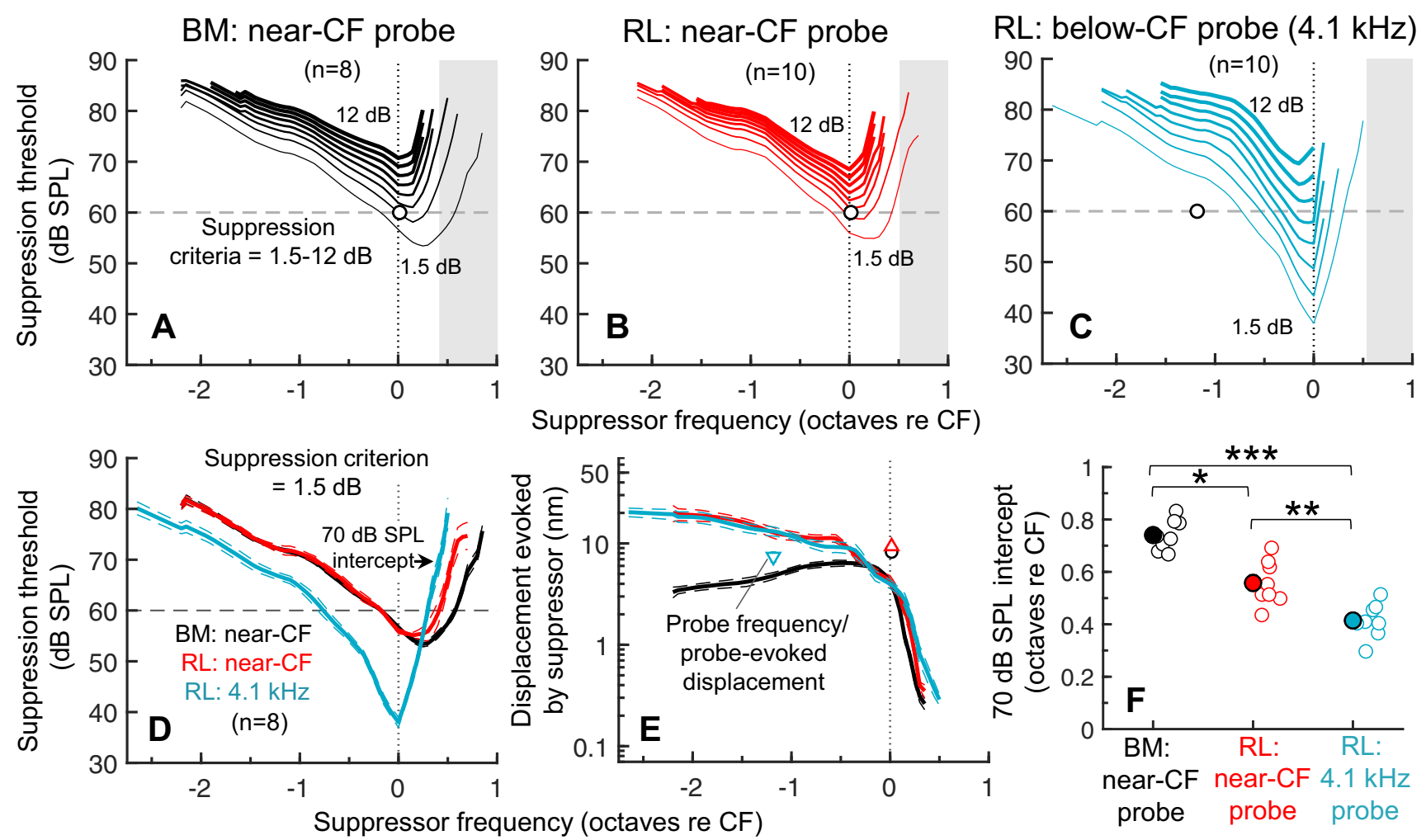

Figure 4. Average suppression thresholds for $B M$ and $R L$ responses to near-CF probes and $R L$ responses to below-CF probes. $\boldsymbol{A}-\boldsymbol{C}$, Average suppressor levels required to suppress $B M(\boldsymbol{A})$ and $R L$ $(B)$ responses to near-CF probes, and RL responses to a $4.1 \mathrm{kHz}$ probe $(\boldsymbol{C})$ by $1.5-12 \mathrm{~dB}$. Because of the different $C$ Fs across preparations ( 9 or $9.5 \mathrm{kHz}$ ), suppressor frequencies are expressed in octaves relative to the $\mathrm{CF}$, and suppression thresholds were interpolated to facilitate averaging. Circles/dashed horizontal lines indicate the probe frequency/level; dotted vertical lines highlight the $\mathrm{CF}$ position. Shaded gray area indicates the range of suppressor frequencies for which the displacement evoked by the suppressor was below the measurement noise floor $(\sim 0.1 \mathrm{~nm})$. Error bars not shown for clarity. D, Comparison of average suppression thresholds (criterion $=1.5 \mathrm{~dB}$ ) from mice in which all three measurement conditions were obtained. Data are shown only at frequencies where suppression thresholds were measurable in at least five mice. Dashed lines indicate \pm SEM. The $70 \mathrm{~dB}$ SPL intercept used to calculate the spatial extent of the buildup region is indicated. $\boldsymbol{E}$, Average displacements evoked by the suppressor tone that reduced each probe response by $1.5 \mathrm{~dB}$. Symbols indicate the frequencies and average displacements elicited by the near-CF and below-CF probe tones when presented alone (error bars are smaller than the symbols). $F$, Comparison of $70 \mathrm{dBSPL}$ intercepts for $1.5 \mathrm{~dB}$ of suppression. Suppression of BM and RL responses to near-CF probes extended to significantly higher suppressor frequencies compared with the suppression of RL responses to a $4.1 \mathrm{kHz}$ probe. BM responses to near-CF probes were also suppressed by significantly higher suppressor frequencies than were RL responses to near-CF probes. Asterisks indicate statistical significance. ${ }^{*} p<0.05,{ }^{* *} p<0.005,{ }^{* * *} p<0.0005$, repeated-measures AN0VA followed by post hoc comparisons with Bonferroni corrections.

shaded regions). For RL responses to the $4.1 \mathrm{kHz}$ probe, small amounts of suppression were most effectively produced by suppressor frequencies near the $\mathrm{CF}$, and suppression was only observed if the suppressor tone produced a displacement at the measurement site (Fig. 3I,L). Amplification of BM and RL responses to near-CF tones therefore built up over some distance basal to the measurement site, whereas the RL response to a 4.1 $\mathrm{kHz}$ tone was amplified locally.

Averaged data $(n=8-10)$ confirmed the different highfrequency extents of suppression for near-CF and $4.1 \mathrm{kHz}$ probes (Fig. 4). To quantitatively compare these extents, we determined the highest-frequency $70 \mathrm{~dB}$ SPL suppressor tone that could suppress each probe response by a criterion amount (Fig. 4D, arrow indicates the $70 \mathrm{~dB}$ SPL intercept for $1.5 \mathrm{~dB}$ of suppression). We found that BM and RL responses to near-CF probes were suppressed by a similar range of suppressor frequencies, though suppression of BM responses extended to slightly higher frequencies for a suppression criterion of $1.5 \mathrm{~dB}$ (Fig. 4D,F; Table 1). This suggests that BM responses depend more strongly than RL responses on amplification provided by locations basal to the measurement site. Importantly, both $\mathrm{BM}$ and $\mathrm{RL}$ responses to near-CF probes were suppressed by significantly higher frequencies than $\mathrm{RL}$ responses to the $4.1 \mathrm{kHz}$ probe, indicating that near-CF responses depended more strongly on amplification from basal locations than did below-CF responses. To estimate the extent of the buildup region for near- $\mathrm{CF}$ and $4.1 \mathrm{kHz}$ responses, we used the frequency-to-place map of the mouse cochlea (Müller et al., 2005) to determine the characteristic location of the $70 \mathrm{~dB}$ SPL intercept frequency, and then calculated its distance from the measurement site. We estimate that amplification built up over a region $0.95 \pm 0.024 \mathrm{~mm}$ basal to the measurement site $(\sim 18 \%$ of the mouse cochlear length, $5.13 \mathrm{~mm}$; Müller et al., 2005) for BM responses to the near-CF probe $(n=$ $8), 0.71 \pm 0.042 \mathrm{~mm}(\sim 14 \%$ of cochlear length) for RL responses to the near-CF probe $(n=10)$, and $0.54 \pm 0.025 \mathrm{~mm}(\sim 10 \%$ of cochlear length) for RL responses to the $4.1 \mathrm{kHz}$ probe $(n=10)$.

Other characteristics of the suppression threshold curves indicate that suppression of RL responses to the $4.1 \mathrm{kHz}$ probe was more strongly tuned to frequencies near the $\mathrm{CF}$, and that these responses were amplified locally (for statistical comparisons, see Table 1). The most effective suppressor frequency (i.e., the frequency at the tip of the suppression threshold curves) for RL responses to the $4.1 \mathrm{kHz}$ probe was lower than those for BM and RL responses to near-CF probes, though only statistically significantly so when compared with BM responses. When compared individually to the CF of the measurement location, suppression threshold tip frequencies were not significantly different from the $\mathrm{CF}$ for $\mathrm{RL}$ responses to the $4.1 \mathrm{kHz}$ probe $\left(t_{(9)}=-1.00, p=\right.$ 
Table 1. Comparison of suppression patterns for near-CF and $4.1 \mathrm{kHz}$ probe tones for each probe level

\begin{tabular}{|c|c|c|c|c|c|}
\hline Probe level, dB SPL & Location, probe frequency & $70 \mathrm{~dB}$ SPL intercept, octaves re CF & $Q_{10 \mathrm{~dB}}$ & Tip frequency, octaves re CF & Tip threshold, dB SPL \\
\hline \multirow[t]{7}{*}{60} & $\mathrm{BM}$, near-CF & $0.74 \pm 0.02$ & $1.51 \pm 0.04$ & $0.27 \pm 0.01$ & $53.28 \pm 0.55$ \\
\hline & $\mathrm{RL}$, near-CF & $0.57 \pm 0.04^{*}$ & $1.94 \pm 0.19^{\text {n.s. }}$ & $0.13 \pm 0.05^{\text {n.s. }}$ & $52.98 \pm 1.03^{\text {n.s. }}$ \\
\hline & $\mathrm{RL}, 4.1 \mathrm{kHz}$ & $0.41 \pm 0.02^{* * * / *}$ & $3.47 \pm 0.18^{* * * / * *}$ & $-0.01 \pm 0.01^{* * * / n . s .}$ & $37.95 \pm 0.98^{* * * * * *}$ \\
\hline & ANOVA & $\begin{array}{l}F_{(2,14)}=33.57 \\
p<0.001, n=8\end{array}$ & $\begin{array}{l}F_{(2,14)}=43.57 \\
p<0.001, n=8\end{array}$ & $\begin{array}{l}F_{(1.00,7.02)}=21.87 \\
p=0.002, n=8\end{array}$ & $\begin{array}{l}F_{(1.12,7.85)}=73.94 \\
p<0.001, n=8\end{array}$ \\
\hline & pairwise & ${ }^{1} p=0.032$ & ${ }^{1} p=0.116$ & ${ }^{1} p=0.092$ & ${ }^{1} p=1.000$ \\
\hline & comparisons & ${ }^{2} p<0.001$ & ${ }^{2} p<0.001$ & ${ }^{2} p<0.001$ & ${ }^{2} p<0.001$ \\
\hline & & ${ }^{3} p=0.011$ & ${ }^{3} p=0.003$ & ${ }^{3} p=0.091$ & ${ }^{3} p<0.001$ \\
\hline \multirow[t]{7}{*}{50} & $\mathrm{BM}$, near-CF & $0.79 \pm 0.03$ & $2.06 \pm 0.22$ & $0.19 \pm 0.05$ & $44.16 \pm 0.67$ \\
\hline & $\mathrm{RL}$, near-CF & $0.83 \pm 0.02^{\text {n.s. }}$ & $2.20 \pm 0.14^{\text {n.s. }}$ & $0.22 \pm 0.03^{\text {n.s. }}$ & $43.78 \pm 0.58^{\text {n.s. }}$ \\
\hline & $\mathrm{RL}, 4.1 \mathrm{kHz}$ & $0.44 \pm 0.04^{* * / * *}$ & $3.47 \pm 0.17^{* * / *}$ & $-0.03 \pm 0.03^{\text {n.s. } / *}$ & $36.13 \pm 1.68^{* / *}$ \\
\hline & ANOVA & $\begin{array}{l}F_{(2,8)}=62.26 \\
p<0.001, n=5\end{array}$ & $\begin{array}{l}F_{(2,10)}=30.79 \\
p<0.001, n=6\end{array}$ & $\begin{array}{l}F_{(2,10)}=15.64 \\
p<0.001, n=6\end{array}$ & $\begin{array}{l}F_{(1.08,5.42)}=21.90 \\
p=0.004, n=6\end{array}$ \\
\hline & pairwise & ${ }^{1} p=1.000$ & ${ }^{1} p=1.000$ & ${ }^{1} p=1.000$ & ${ }^{1} p=1.000$ \\
\hline & comparisons & ${ }^{2} p=0.003$ & ${ }^{2} p=0.001$ & ${ }^{2} p=0.052$ & ${ }^{2} p=0.008$ \\
\hline & & ${ }^{3} p=0.001$ & ${ }^{3} p=0.006$ & ${ }^{3} p=0.012$ & ${ }^{3} p=0.024$ \\
\hline \multirow[t]{3}{*}{40} & $\mathrm{BM}$, near-CF & $0.97 \pm 0.03$ & $2.68 \pm 0.17$ & $0.15 \pm 0.002$ & $34.50 \pm 0.54$ \\
\hline & $\mathrm{RL}$, near-CF & $0.91 \pm 0.04^{\text {n.s. }}$ & $2.49 \pm 0.22^{\text {n.s. }}$ & $0.17 \pm 0.02^{\text {n.s. }}$ & $35.25 \pm 0.47^{\text {n.s. }}$ \\
\hline & Paired $t$ test & $t_{(4)}=1.32, p=0.258, n=5$ & $t_{(5)}=1.42, p=0.214, n=6$ & $t_{(5)}=-1.00, p=0.363, n=6$ & $t_{(5)}=-1.71, p=0.148, n=6$ \\
\hline
\end{tabular}

Summary of suppression threshold curve parameters (mean \pm SEM) for all mice in which data for each measurement condition (BM, near-CF probe; RL, near-CF probe; RL, $4.1 \mathrm{kHz}$ probe) were available for a given probe level. All parameter values were derived from the suppression threshold curves for a suppression criterion of $1.5 \mathrm{~dB}$. For the RL, near-CF and RL, $4.1 \mathrm{kHz}$ probe conditions at each probe level, asterisks indicate statistically significant differences in parameter values with respect to the BM, near-CF probe condition. ${ }^{*} p<0.05,{ }^{*} p<0.005,{ }^{* * *} p<0.0005 ; n . s$. $=$ non-statistically significant. For the RL, $4.1 \mathrm{kHz}$ probe condition, a second set of asterisks indicates a statistically significant difference with respect to the RL, near-CF probe condition. Differences were assessed by repeated-measures ANOVA, followed by post hoc pairwise comparisons with Bonferroni corrections, for probe levels of $50-60 \mathrm{~dB} \mathrm{SPL}$, and by paired $t$ test for a probe level of $40 \mathrm{~dB} \mathrm{SPL}$, as data were not obtained for the RL, $4.1 \mathrm{kHz}$ probe condition at this probe level. Results of the relevant statistical analyses are shown along with the number of mice $(n)$ included in each average value. When repeated-measures ANOVAs were performed, the $p$ values for each pairwise comparison (indicated by superscript numbers: ${ }^{1} \mathrm{BM}$, near-CF versus RL, near-CF; ${ }^{2} \mathrm{BM}$, near-CF versus $\mathrm{RL}, 4.1 \mathrm{kHz}$; ${ }^{3} \mathrm{RL}$, near- $\mathrm{CF}$ vs $\mathrm{RL}, 4.1 \mathrm{kHz}$ ) are shown.

0.343, $n=10$, one-sample $t$ test), whereas they were significantly higher than the $\mathrm{CF}$ for both $\mathrm{BM}$ and $\mathrm{RL}$ responses to near-CF probes $\left(\mathrm{BM}: t_{(7)}=30.02, p<0.001, n=8\right.$; $\mathrm{RL}: t_{(9)}=3.93, p=0.003, n=10$, onesample $t$ test). The suppression of RL responses to the $4.1 \mathrm{kHz}$ probe was also more sharply tuned, as quantified by the quality factor, $\mathrm{Q}_{10 \mathrm{~dB}}$. Lastly, suppression of RL responses to the $4.1 \mathrm{kHz}$ probe required significantly lower suppressor levels, particularly at frequencies near the CF. This may reflect that amplification of the $4.1 \mathrm{kHz}$ response was strongly localized to the measurement site, rather than being distributed over a basal region. Alternatively, near-CF probes may exert more of a suppressive influence on the response to the suppressor tones, thus reducing their efficacy.

\section{Amplification of near-CF responses builds up over a broader region at lower stimulus levels}

We initially studied the suppression of responses to probe tones presented at $60 \mathrm{~dB}$ $\mathrm{SPL}$, as BM and RL responses to near-CF tones and $\mathrm{RL}$ responses to $4.1 \mathrm{kHz}$ tones were comparable in magnitude at this level (BM, near-CF: $8.24 \pm 0.51 \mathrm{~nm}$; RL, near-CF: $9.35 \pm 0.59 \mathrm{~nm}, \mathrm{RL}, 4.1 \mathrm{kHz}$ : $7.41 \pm 0.83 \mathrm{~nm}$; no significant pairwise differences after repeated-measures ANOVA, $\left.F_{(2,14)}=4.29, p=0.035, n=8\right)$. However, because both the probe and suppressor tones contribute to the saturation of $\mathrm{OHC}$

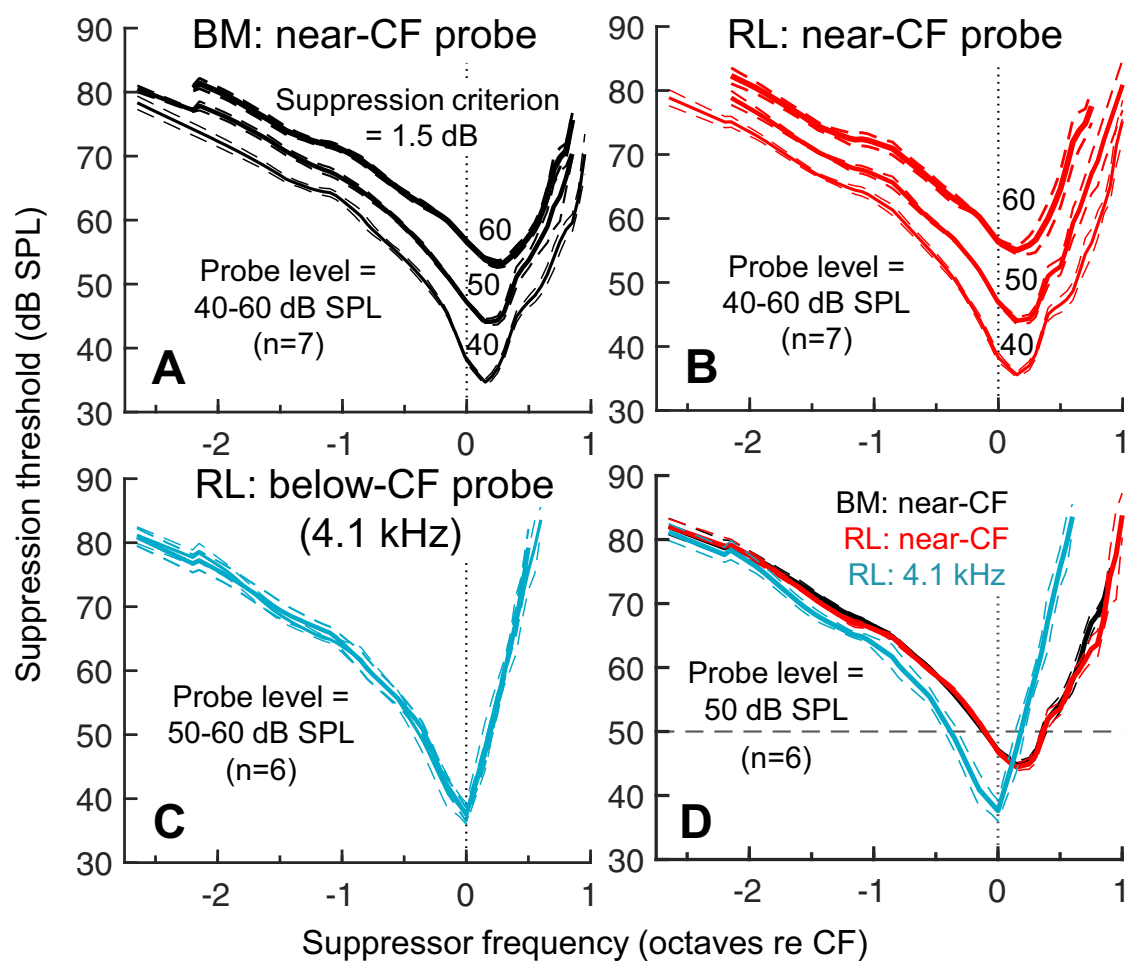

Figure 5. Suppression extends to higher suppressor frequencies with decreasing probe level for near- $C F$, but not below$(\mathrm{C}$, probes. $\boldsymbol{A}, \boldsymbol{B}$, Average suppression thresholds (criterion $=1.5 \mathrm{~dB}$ ) for $\mathrm{BM}(\boldsymbol{A})$ and $\mathrm{RL}(\boldsymbol{B})$ responses to near- $\mathrm{CF}$ probe tones presented at levels of 40,50, and $60 \mathrm{~dB}$ SPL. With decreasing probe level, suppression was observed at lower suppressor levels and higher suppressor frequencies, indicating a broadening of the buildup region. Averages include data from mice in which suppression was characterized for all three probe levels, and are shown only at frequencies where suppression criteria were met in at least five mice. C, Average suppression thresholds (criterion $=1.5 \mathrm{~dB}$ ) for RL responses to a $4.1 \mathrm{kHz}$ probe presented at 50 and $60 \mathrm{~dB}$ SPL. Suppression thresholds for the two probe levels were highly similar. Average data inclusion/plotting criteria are as described above. $D$, Comparison of average suppression thresholds for $50 \mathrm{~dB}$ SPL probes. Dashed horizontal line indicates the probe level. For all curves in $\boldsymbol{A}-\boldsymbol{D}$, dashed lines indicate \pm SEM. Dotted vertical lines highlight the $C F$ position. 


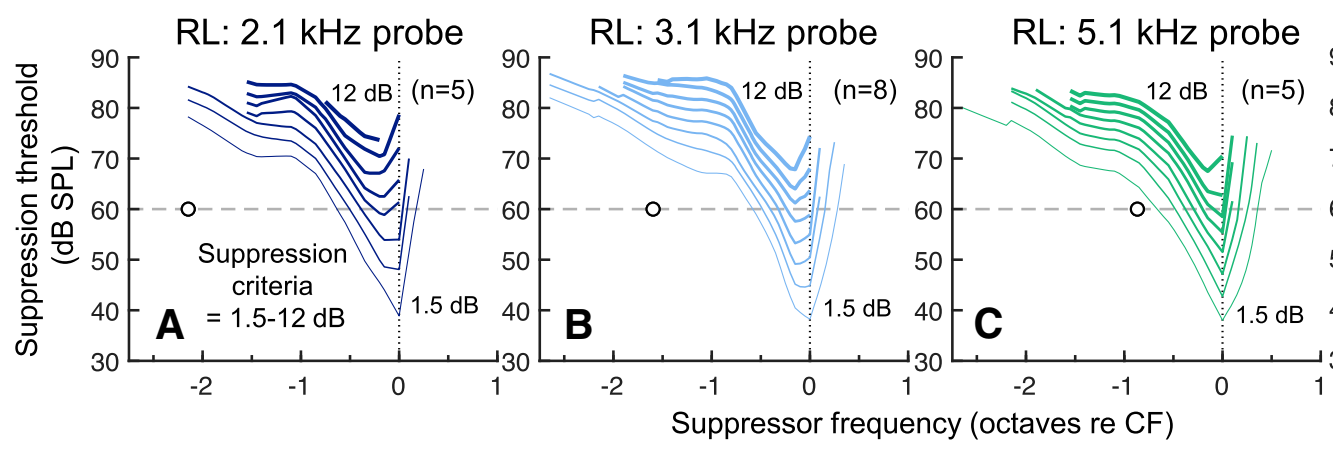

RL: $6.1 \mathrm{kHz}$ probe

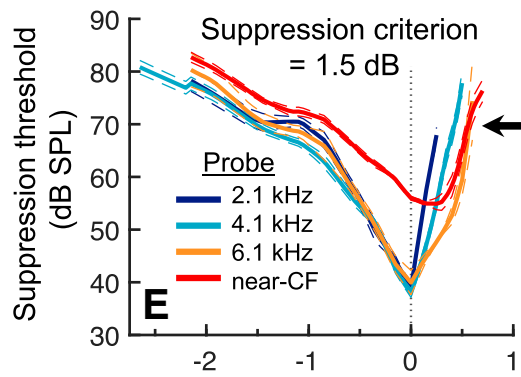

Suppressor frequency (octaves re CF)

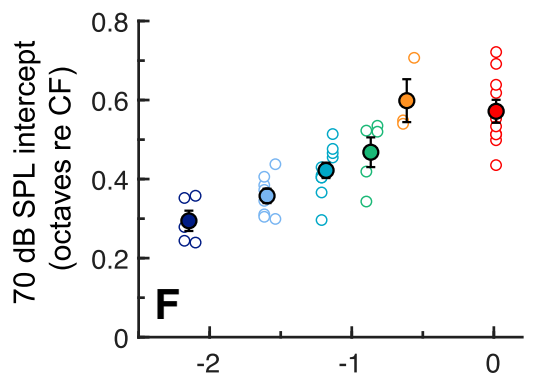

Probe frequency (octaves re CF)

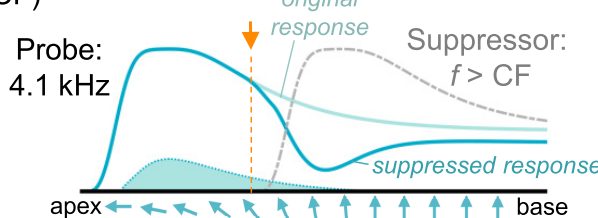

G

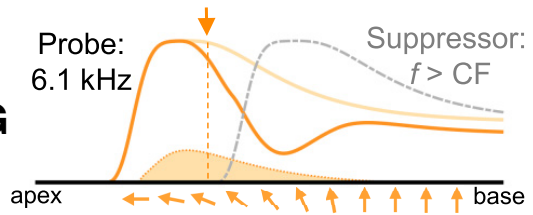

Figure 6. Suppression of RL responses to different below-CF probes reveals a gradual transition between the region of the RL traveling wave where amplification builds up and where it is primarily local. $\boldsymbol{A}-\boldsymbol{D}$, Average suppression thresholds for RL responses to probe frequencies of 2.1,3.1,5.1, and $6.1 \mathrm{kHz}$ ( $\boldsymbol{A}-\boldsymbol{D}$, respectively) for a probe level of $60 \mathrm{~dB}$ SPL. Circles/dashed horizontal lines indicate the probe frequency and level; dotted vertical lines highlight the CF position. Averaged data are only shown at frequencies where the suppression criterion was met and displacements were above the noise floor in at least five mice (when $n=8$ ), four mice (when $n=5$ ), or in three mice (when $n=3$ ). Error bars not shown for clarity. $\boldsymbol{E}$, Comparison of average suppression thresholds (criterion $=1.5 \mathrm{~dB}$ ) for RL responses to 2.1, 4.1, and $6.1 \mathrm{kHz}$ probes, as well as a near-CF probe. Dashed lines indicate \pm SEM. $F$, Comparison of the $70 \mathrm{~dB}$ SPL intercept of the suppression threshold curves (indicated by arrow in $\boldsymbol{E}$ ) for all probe frequencies. With decreasing probe frequency, above-CF suppressor tones became progressively less effective at suppressing the probe response, indicating that the buildup region became increasingly narrow. Individual/average data are shown with open/filled symbols. Error bars indicate \pm SEM. G, Diagram illustrating that the data suggest a smooth transition between the region of the traveling wave where amplification builds up (apically-pointing arrows), and where amplification is primarily local (vertical arrows). The position of our measurement site (orange arrows/vertical dashed lines) in this transition region explains the increased effectiveness of an above-CF suppressor tone (dashed gray lines) in suppressing the response to a $6.1 \mathrm{kHz}$ versus a $4.1 \mathrm{kHz}$ probe tone. The passive response to each probe tone is also shown (dotted curves/shaded regions).

mechanotransduction currents, the probe level may influence the observed suppression patterns (Rhode, 2007). Moreover, the spatial extent of the buildup region may depend on stimulus level. We therefore next confirmed that the differences in suppression patterns noted above were observed at lower probe levels.

As we decreased the probe level from 60 to 50 and then $40 \mathrm{~dB}$ SPL, the suppression of BM and RL responses to near-CF probes was observed at lower suppressor levels and higher suppressor frequencies (Fig. $5 A, B$ ). The probe level had a significant effect on the $70 \mathrm{~dB}$ SPL suppression threshold intercept $\left(\mathrm{BM}: F_{(2,8)}=\right.$ 35.99, $p<0.001, n=5$; RL: $F_{(2,10)}=16.41, p<0.001, n=6$, repeated-measures ANOVA), with the $70 \mathrm{~dB}$ SPL intercept frequency being significantly higher for a probe level of $40 \mathrm{~dB}$ SPL versus $60 \mathrm{~dB}$ SPL (BM: $p=0.007$; RL: $p=0.003$ ). Amplification of near-CF responses therefore built up over a broader basal region at lower probe levels. Using the $70 \mathrm{~dB}$ SPL intercepts of the suppression threshold curves (and suppression criterion $=1.5$ $\mathrm{dB}$ ), we estimate that the buildup region for 50 and $40 \mathrm{~dB}$ SPL probe responses extended $1.02 \pm 0.029 \mathrm{~mm}(n=7)$ and $1.23 \pm$ $0.033 \mathrm{~mm}(n=5)$ basal for the BM, and $1.03 \pm 0.051 \mathrm{~mm}(n=8)$ and $1.16 \pm 0.038 \mathrm{~mm}(n=6)$ basal for the RL.

In contrast, the suppression of RL responses to a $4.1 \mathrm{kHz}$ probe did not change as the probe level was reduced from 60 to 50 dB SPL (Fig. 5C; comparison of $70 \mathrm{~dB}$ SPL intercepts: $t_{(4)}=0.003$, $p=0.998, n=5$, paired $t$ test), with an estimated buildup region of $0.56 \pm 0.052 \mathrm{~mm}$ at $50 \mathrm{~dB}$ SPL $(n=5$; responses at $40 \mathrm{~dB}$ SPL were too low in amplitude to characterize suppression). Differ- ences in the high-frequency extents of suppression for $4.1 \mathrm{kHz}$ versus near-CF probes were therefore slightly larger for a probe level of $50 \mathrm{~dB}$ SPL (Fig. 5D) than for a probe level of $60 \mathrm{~dB}$ SPL (Fig. 4D). For both probe levels, suppression threshold curves for RL responses to a $4.1 \mathrm{kHz}$ probe had lower-frequency $70 \mathrm{~dB}$ SPL intercepts and lower tip thresholds, and were more sharply tuned than those for BM or RL responses to near-CF probes (Table 1). Additionally, suppression patterns for BM and RL responses to near-CF tones became more similar at lower probe levels, suggesting that the BM and RL may, in fact, share a common buildup region (70 dB SPL intercepts were not significantly different at probe levels of either 50 or $40 \mathrm{~dB}$ SPL; Table 1).

\section{The buildup region of the RL traveling wave gradually} transitions into the region where amplification is local Differences in the extent of the buildup regions for near-CF and $4.1 \mathrm{kHz}$ tones indicate that RL motion distant from the traveling-wave peak is locally amplified. However, above-CF suppressors could still produce $\sim 1.5-3 \mathrm{~dB}$ of suppression of the $4.1 \mathrm{kHz}$ response even when they evoked very little displacement of the BM or RL (Figs. $3 L, 4 E$ ). These effects were likely driven by suppression of $\mathrm{OHC}$ activity at locations slightly basal, suggesting that our measurement site fell in a region where there was still some spatial buildup of amplification for the 4.1 $\mathrm{kHz}$ response. We therefore next examined whether the degree of buildup depended on the distance of our measurement site from the peak of the traveling wave, which we varied by changing the probe frequency. 
We found that the suppression of RL responses to below-CF probe frequencies ranging from 2.1 to $6.1 \mathrm{kHz}$ generally resembled that observed for the $4.1 \mathrm{kHz}$ probe (Fig. 6A-E). However, with increasing probe frequency, above-CF suppressor tones became progressively more effective at reducing the response to the probe. This was evidenced by increases in the frequency of the 70 $\mathrm{dB}$ SPL suppression threshold intercept as the probe frequency was increased (Fig. 6E, F; $F_{(3,12)}=7.48, p=0.004, n=5$, repeated-measures ANOVA for probe frequencies between 2.1 and $5.1 \mathrm{kHz} ; 6.1 \mathrm{kHz}$ probe data excluded because of low $n$ ). Above-CF suppressor tones were, in fact, as effective in reducing the response to a $6.1 \mathrm{kHz}$ probe as they were for a near-CF probe $\left(t_{(2)}=1.06, p=0.401, n=3\right.$, paired-samples $t$ test). We estimate that the buildup region extended $0.76 \pm 0.069 \mathrm{~mm}(n=3)$ basal to the measurement site for the $6.1 \mathrm{kHz}$ probe, but only $0.38 \pm$ $0.033 \mathrm{~mm}(n=5)$ for the $2.1 \mathrm{kHz}$ probe. Thus, the degree to which the RL response accumulated gain from more basal regions depended on the proximity of our measurement site to the peak of the traveling wave. The region of the wave where amplification is primarily local (further from the peak) appears to smoothly transition into the region where amplification builds up significantly (closer to the peak; Fig. 6G). The buildup of amplification is therefore likely controlled by a property that varies continuously along the length of the cochlea, or that varies with position in the traveling-wave vibration pattern.

\section{Discussion}

It is well established that the BM traveling wave is selectively amplified near its peak and accumulates gain over the amplifying region (Fisher et al., 2012). Here we demonstrate that although the RL traveling wave is amplified throughout its basal extent, it too only accumulates gain near the wave's peak. Elsewhere, $\mathrm{OHC}$-generated forces locally amplify RL motion without influencing RL (or BM) motion at neighboring locations. This contradicts the proposition that amplification of below-CF RL motion underlies a global mechanism for amplifying the traveling-wave peak (Ren et al., 2016b; He et al., 2018). Such a mechanism was proposed based on the observation of OHCmediated, anti-phasic RL and BM motion at below-CF frequencies in the cochlear base of mouse and gerbil. This motion could theoretically pump fluid longitudinally along the organ of Corti to enhance the traveling wave as it propagates apically (Karavitaki and Mountain, 2007; Zagadou and Mountain, 2012). However, our measurements indicate that $\mathrm{OHC}$ activity in the base contributes little to the amplification of BM or RL traveling waves in the apex. Instead, our data reveal that a spatially-selective mechanism controls the buildup of amplification along both the bottom and top of the organ of Corti.

While the buildup of amplification is necessary to produce sensitive responses at the traveling-wave peak, restricting its spatial extent is also advantageous. Such restriction sharpens the wave's peak, thereby enhancing frequency selectivity, and ensures that cochlear sensitivity at each location depends on a limited population of nearby OHCs. This prevents high-frequency sounds from interfering with the amplification of low-frequency sounds, which likely aids in processing broadband signals such as speech and environmental sounds. Importantly, this restriction also minimizes the impact of OHC damage in one location on sensitivity elsewhere, such that $\mathrm{OHC}$ damage in the cochlear base has little effect on apical responses (Dallos and Harris, 1978; Cody, 1992).

Though the buildup region for near-CF tones appears to be slightly broader for the $\mathrm{BM}(\sim 0.95-1.23 \mathrm{~mm})$ than the $\mathrm{RL}$
( $\sim 0.71-1.16 \mathrm{~mm}$ ), the buildup of amplification along the BM and RL is likely controlled by common mechanisms (as discussed below). The broader buildup region observed for the BM versus $\mathrm{RL}$ response to a $60 \mathrm{~dB} S P L$ probe can be explained by the fact that above-CF suppressors reduce the gain provided by basal locations without directly stimulating the measurement site, and thus effectively reduce the local probe level (Versteegh and van der Heijden, 2013). Because RL vibrations grow more compressively than $\mathrm{BM}$ vibrations with increasing level, reducing the local probe level has less of an effect on RL responses to moderate-level probes. Likewise, the broadening of the buildup region with decreasing probe level can be explained by the less compressive growth of near-CF BM and RL responses at lower stimulus levels. Reducing the local probe level therefore impacts a $40 \mathrm{~dB}$ SPL response more than a $60 \mathrm{~dB}$ SPL response. The buildup region's apparent extent thus partially reflects whether local amplification can compensate for the loss of amplification provided by basal locations.

Although we refer to the buildup region as being spatially restricted, $0.71-1.23 \mathrm{~mm}$ spans a considerable portion of the mouse's cochlear length ( $\sim 14-24 \%$ of $5.13 \mathrm{~mm}$; Müller et al., 2005). Similar absolute extents of the buildup region can be estimated from BM suppression data in chinchilla $(\sim 1.8 \mathrm{~mm}$; Rhode, 2007) and gerbil ( 1.1-1.3 mm; Versteegh and van der Heijden, 2013), though these distances only span $\sim 9-10 \%$ of the cochlear length $(\sim 20.1$ and $11.1 \mathrm{~mm}$ in chinchilla and gerbil, respectively; Müller, 1996; Müller et al., 2010). Similar or slightly shorter buildup regions have been estimated from the effects of inactivating $\mathrm{OHC}$ motility on $\mathrm{BM}$ vibrations in chinchilla $(>0.5$ $\mathrm{mm}$; Fisher et al., 2012) and the effects of acoustic trauma on auditory nerve fiber sensitivity in guinea pig $(\sim 0.5-1.3 \mathrm{~mm}$; Cody, 1992). Thus, although cochlear length may vary widely across species, the absolute longitudinal influence of OHC activity on cochlear vibrations appears to be relatively constant.

The amplification of below-CF RL motion indicates that $\mathrm{OHCs}$ are stimulated over a wide frequency range and provide wideband force generation in response (Frank et al., 1999; Ren et al., 2016a). Whereas the BM does not respond to these below-CF forces, the RL does, likely because it is directly attached to the apical surfaces of the OHCs and is less stiff than the BM (Mammano and Ashmore, 1993; Richter and Quesnel, 2006). However, whether these forces build up longitudinally depends on the degree of coupling between adjacent locations. Our findings indicate that direct coupling of $\mathrm{OHC}$-generated forces along the $\mathrm{RL}$ is weak, because there was little spatial buildup of below-CF RL amplification. Instead, our data suggest that longitudinal coupling of OHC-generated forces may be largely mediated by the $\mathrm{BM}$ and its interactions with the cochlear fluids. This is because the buildup of RL amplification was only observed near the peak of the traveling wave, where OHC-generated forces are capable of influencing BM motion. If the BM's spatially-graded impedance underlies how OHCs selectively amplify BM motion near the peak of the traveling wave, then this property could also explain why longitudinal coupling is limited to this region.

However, the micromechanics of the organ of Corti are likely also involved in spatially restricting BM amplification and/or the longitudinal coupling of OHC-generated forces. For instance, maximal BM amplification occurs when $\mathrm{OHC}$ force generation is in phase with $\mathrm{BM}$ velocity, such that a frequency-dependence in the phase of the $\mathrm{OHC}$ stimulation (and, thus, force production) could produce selective BM amplification near the CF (Dong and Olson, 2013; Nankali et al., 2018). The Y-shaped geometry formed longitudinally by the OHCs and Deiters' cells has also 
been proposed to underlie the spatially-selective amplification and buildup of BM motion (Geisler and Sang, 1995; Yoon et al., 2011). A model that emphasizes the importance of this geometry has recently been extended to include the RL's mechanics and replicates some of the differences in RL and BM motion (Motallebzadeh et al., 2018). The similarity in the buildup region's extent across species supports the idea that longitudinal coupling of OHC-generated forces depends on the cellular dimensions and geometry within the organ of Corti, which are also similar across species (Yoon et al., 2011).

Additionally, although longitudinal coupling along the apical surface of the organ of Corti is apparently weak below the CF, it is possible that this coupling is enhanced near the CF. For instance, the tectorial membrane (TM) and its interactions with OHC stereocilia have been shown to confer longitudinal coupling to the cochlear partition and increase near-CF responses (Russell et al., 2007; Dewey et al., 2018). This coupling likely involves longitudinally-propagating waves of radial motion on the TM (Ghaffari et al., 2007; Sellon et al., 2015). Because the micromechanics of the organ of Corti enhance radial RL motion near the CF (Lee et al., 2016), the interplay between radial RL and TM motion could facilitate longitudinal coupling of OHC-generated forces at these frequencies. Longitudinal electrical coupling between OHCs (Ramamoorthy et al., 2007) could also be frequency dependent.

The functional role of amplifying below-CF RL motion remains uncertain. Nevertheless, this motion must shape how the OHCs and IHCs are stimulated, because their stereocilia protrude from the RL. As evidence for this, below-CF auditory nerve fiber responses can be inhibited by tones (Nam and Guinan, 2018) and by stimulation of the medial olivocochlear efferents (Stankovic and Guinan, 1999, 2000), which innervate OHCs. This suggests that IHCs are excited by the amplified, below-CF RL motion. However, IHC receptor potentials are relatively insensitive to below-CF tones (Russell et al., 1986) compared with RL vibrations, and auditory nerve fiber tuning more closely resembles the BM's mechanical tuning in the cochlear base (Narayan et al., 1998). The influence of below-CF RL motion may therefore be filtered out of the mechanical drive to the IHC stereocilia or by the mechanotransduction process. Sensitivity to velocity, rather than displacement, (Robles and Ruggero, 2001) or fast adaptation of mechanotransduction currents (Kennedy et al., 2003; Ricci et al., 2005) could provide this high-pass filtering.

OHCs are more sensitive than IHCs to below-CF tones (Russell et al., 1986; Kössl and Russell, 1992), perhaps reflecting a greater influence of RL motion. Additionally, the suppression of below-CF RL motion may have correlates in the suppression of other OHC-dependent responses, such as the cochlear microphonic (Legouix et al., 1973; Cheatham and Dallos, 1982) and otoacoustic emissions (Martin et al., 1999; Charaziak and Siegel, 2015), by tones presented several octaves above the stimulus frequency. Nevertheless, the semitransverse RL displacements reported here are likely a poor estimate of the stimulus to the OHC stereocilia, which are deflected by radial shear between the RL and TM. Future studies of 3-D cochlear micromechanics are needed to fully understand how RL motion influences the radial stimulation of hair cells, and thus the auditory nerve. Here we show that the influence of transverse RL motion along the longitudinal direction is spatially restricted by mechanisms that likely derive from the mechanical properties of the BM and organ of Corti.

\section{References}

Ashmore J, Avan P, Brownell WE, Dallos P, Dierkes K, Fettiplace R, Grosh K, Hackney CM, Hudspeth AJ, Jülicher F, Lindner B, Martin P, Meaud J, Petit C, Santos-Sacchi JR, Sacchi JR, Canlon B (2010) The remarkable cochlear amplifier. Hear Res 266:1-17.

Békésy von G (1960) Experiments in hearing. New York: McGraw-Hill.

Charaziak KK, Siegel JH (2015) Tuning of SFOAEs evoked by lowfrequency tones is not compatible with localized emission generation. J Assoc Res Otolaryngol 16:317-329.

Cheatham MA, Dallos P (1982) Two-tone interactions in the cochlear microphonic. Hear Res 8:29-48.

Cody AR (1992) Acoustic lesions in the mammalian cochlea: implications for the spatial distribution of the "active process". Hear Res 62:166-172.

Cooper NP (1996) Two-tone suppression in cochlear mechanics. J Acoust Soc Am 99:3087-3098.

Cooper NP, Vavakou A, van der Heijden M (2018) Vibration hotspots reveal longitudinal funneling of sound-evoked motion in the mammalian cochlea. Nat Commun 9:3054.

Dallos P, Harris D (1978) Properties of auditory nerve responses in absence of outer hair cells. J Neurophysiol 41:365-383.

Dewey JB, Xia A, Müller U, Belyantseva IA, Applegate BE, Oghalai JS (2018) Mammalian auditory hair cell bundle stiffness affects frequency tuning by increasing coupling along the length of the cochlea. Cell Rep 23:29152927.

Dong W, Olson ES (2013) Detection of cochlear amplification and its activation. Biophys J 105:1067-1078.

Fisher JA, Nin F, Reichenbach T, Uthaiah RC, Hudspeth AJ (2012) The spatial pattern of cochlear amplification. Neuron 76:989-997.

Frank G, Hemmert W, Gummer AW (1999) Limiting dynamics of highfrequency electromechanical transduction of outer hair cells. Proc Natl Acad Sci U S A 96:4420-4425.

Gao SS, Wang R, Raphael PD, Moayedi Y, Groves AK, Zuo J, Applegate BE, Oghalai JS (2014) Vibration of the organ of Corti within the cochlear apex in mice. J Neurophysiol 112:1192-1204.

Geisler CD, Sang C (1995) A cochlear model using feed-forward outer-haircell forces. Hear Res 86:132-146.

Geisler CD, Yates GK, Patuzzi RB, Johnstone BM (1990) Saturation of outer hair cell receptor currents causes two-tone suppression. Hear Res 44:241256.

Ghaffari R, Aranyosi AJ, Freeman DM (2007) Longitudinally propagating traveling waves of the mammalian tectorial membrane. Proc Natl Acad Sci U S A 104:16510-16515.

He W, Kemp D, Ren T (2018) Timing of the reticular lamina and basilar membrane vibration in living gerbil cochleae. eLife 7:e37625.

Karavitaki KD, Mountain DC (2007) Evidence for outer hair cell driven oscillatory fluid flow in the tunnel of corti. Biophys J 92:3284-3293.

Keiler S, Richter CP (2001) Cochlear dimensions obtained in hemicochleae of four different strains of mice: CBA/CaJ, 129/CD1, 129/SvEv and C57BL/6J. Hear Res 162:91-104.

Kennedy HJ, Evans MG, Crawford AC, Fettiplace R (2003) Fast adaptation of mechanoelectrical transducer channels in mammalian cochlear hair cells. Nat Neurosci 6:832-836.

Kössl M, Russell IJ (1992) The phase and magnitude of hair cell receptor potentials and frequency tuning in the guinea pig cochlea. J Neurosci 12:1575-1586.

Lee HY, Raphael PD, Park J, Ellerbee AK, Applegate BE, Oghalai JS (2015) Noninvasive in vivo imaging reveals differences between tectorial membrane and basilar membrane traveling waves in the mouse cochlea. Proc Natl Acad Sci U S A 112:3128-3133.

Lee HY, Raphael PD, Xia A, Kim J, Grillet N, Applegate BE, Ellerbee Bowden AK, Oghalai JS (2016) Two-dimensional cochlear micromechanics measured in vivo demonstrate radial tuning within the mouse organ of corti. J Neurosci 36:8160-8173.

Legouix JP, Remond MC, Greenbaum HB (1973) Interference and twotone inhibition. J Acoust Soc Am 53:409-419.

Mammano F, Ashmore JF (1993) Reverse transduction measured in the isolated cochlea by laser michelson interferometry. Nature 365:838-841.

Martin GK, Stagner BB, Jassir D, Telischi FF, Lonsbury-Martin BL (1999) Suppression and enhancement of distortion-product otoacoustic emissions by interference tones above $f_{2}$ : I. basic findings in rabbits. Hear Res 136:105-123.

Motallebzadeh H, Soons JAM, Puria S (2018) Cochlear amplification and 
tuning depend on the cellular arrangement within the organ of corti. Proc Natl Acad Sci U S A 115:5762-5767.

Müller M (1996) The cochlear place-frequency map of the adult and developing Mongolian gerbil. Hear Res 94:148-156.

Müller M, von Hünerbein K, Hoidis S, Smolders JW (2005) A physiological place-frequency map of the cochlea in the CBA/J mouse. Hear Res 202:63-73.

Müller M, Hoidis S, Smolders JW (2010) A physiological frequencyposition map of the chinchilla cochlea. Hear Res 268:184-193.

Nam H, Guinan JJ Jr (2018) Non-tip auditory-nerve responses that are suppressed by low-frequency bias tones originate from reticular lamina motion. Hear Res 358:1-9.

Nankali A, Wang Y, Olson ES, Grosh K (2018) Frequency structure in intracochlear voltage supports the concept of tectorial membrane mechanical resonance. In: To the ear and back again, advances in auditory biophysics, Proceedings of the 13th Mechanics of Hearing Workshop (Bergevin C, Puria S, eds), p 040002. Melville, NY: AIP.

Narayan SS, Temchin AN, Recio A, Ruggero MA (1998) Frequency tuning of basilar membrane and auditory nerve fibers in the same cochleae. Science 282:1882-1884.

Oghalai JS (2004) the cochlear amplifier: augmentation of the traveling wave within the inner ear. Curr Opin Otolaryngol Head Neck Surg 12:431-438.

Patuzzi R, Robertson D (1988) Tuning in the mammalian cochlea. Physiol Rev 68:1009-1082.

Ramamoorthy S, Deo NV, Grosh K (2007) A mechano-electro-acoustical model for the cochlea: response to acoustic stimuli. J Acoust Soc Am 121:2758-2773.

Ren T (2002) Longitudinal pattern of basilar membrane vibration in the sensitive cochlea. Proc Natl Acad Sci U S A 99:17101-17106.

Ren T, He W, Barr-Gillespie PG (2016a) Reverse transduction measured in the living cochlea by low-coherence heterodyne interferometry. Nat Commun 7:10282.

Ren T, He W, Kemp D (2016b) Reticular lamina and basilar membrane vibrations in living mouse cochleae. Proc Natl Acad Sci USA 113:9910-9915.

Rhode WS (2007) Mutual suppression in the $6 \mathrm{kHz}$ region of sensitive chinchilla cochleae. J Acoust Soc Am 121:2805-2818.

Rhode WS, Recio A (2000) Study of mechanical motions in the basal region of the chinchilla cochlea. J Acoust Soc Am 107:3317-3332.

Ricci AJ, Kennedy HJ, Crawford AC, Fettiplace R (2005) The transduction channel filter in auditory hair cells. J Neurosci 25:7831-7839.
Richter CP, Quesnel A (2006) Stiffness properties of the reticular lamina and the tectorial membrane as measured in the gerbil cochlea. In: Auditory mechanisms processes and models (Nuttall AL, Ren T, Gillespie P, Grosh K, de Boer E, eds), pp 70-78. Hackensack, NJ: World Scientific.

Robles L, Ruggero MA (2001) Mechanics of the mammalian cochlea. Physiol Rev 81:1305-1352.

Ruggero MA, Robles L, Rich NC (1992) Two-tone suppression in the basilar membrane of the cochlea: mechanical basis of auditory-nerve rate suppression. J Neurophysiol 68:1087-1099.

Russell IJ, Nilsen KE (1997) The location of the cochlear amplifier: spatial representation of a single tone on the guinea pig basilar membrane. Proc Natl Acad Sci U S A 94:2660-2664.

Russell IJ, Cody AR, Richardson GP (1986) The responses of inner and outer hair cells in the basal turn of the guinea-pig cochlea and in the mouse cochlea grown in vitro. Hear Res 22:199-216.

Russell IJ, Legan PK, Lukashkina VA, Lukashkin AN, Goodyear RJ, Richardson GP (2007) Sharpened cochlear tuning in a mouse with a genetically modified tectorial membrane. Nat Neurosci 10:215-223.

Sellon JB, Farrahi S, Ghaffari R, Freeman DM (2015) Longitudinal spread of mechanical excitation through tectorial membrane traveling waves. Proc Natl Acad Sci U S A 112:12968-12973.

Soons JA, Ricci AJ, Steele CR, Puria S (2015) Cytoarchitecture of the mouse organ of Corti from base to apex, determined using in situ two-photon imaging. J Assoc Res Otolaryngol 16:47-66.

Stankovic KM, Guinan JJ Jr (1999) Medial efferent effects on auditorynerve responses to tail-frequency tones: I. rate reduction. J Acoust Soc Am 106:857-869.

Stankovic KM, Guinan JJ Jr (2000) Medial efferent effects on auditorynerve responses to tail-frequency tones II: alteration of phase. J Acoust Soc Am 108:664-678.

Versteegh CP, van der Heijden M (2013) The spatial buildup of compression and suppression in the mammalian cochlea. J Assoc Res Otolaryngol 14:523-545.

Yoon YJ, Steele CR, Puria S (2011) Feed-forward and feed-backward amplification model from cochlear cytoarchitecture: an interspecies comparison. Biophys J 100:1-10.

Zagadou BF, Mountain DC (2012) Analysis of the cochlear amplifier fluid pump hypothesis. J Assoc Res Otolaryngol 13:185-197.

Zajic G, Schacht J (1987) Comparison of isolated outer hair cells from five mammalian species. Hear Res 26:249-256. 